\title{
Gelation as arrested phase separation in short-ranged attractive colloid-polymer mixtures
}

\author{
Emanuela Zaccarelli ${ }^{1,2}$, Peter J. Lu ${ }^{3}$, Fabio Ciulla ${ }^{1}$, David. A. \\ Weitz $^{3}$ and Francesco Sciortino ${ }^{1,2}$ \\ ${ }^{1}$ Dipartimento di Fisica and ${ }^{2}$ CNR-INFM-SOFT, Università di Roma La Sapienza, \\ P.le A. Moro 2, I-00185 Rome, Italy \\ ${ }^{3}$ Department of Physics, and SEAS, Harvard University, Cambridge, MA 02138, USA \\ E-mail: emanuela.zaccarelli@phys.uniroma1.it, plu@fas.harvard.edu
}

\begin{abstract}
We present further evidence that gelation is an arrested phase separation in attractive colloid-polymer mixtures, based on a method combining confocal microscopy experiments with numerical simulations recently established in Nature 453, 499 (2008). Our results are independent of the form of the interparticle attractive potential, and therefore should apply broadly to any attractive particle system with short-ranged, isotropic attractions. We also give additional characterization of the gel states in terms of their structure, inhomogeneous character and local density.
\end{abstract}

\section{Introduction}

The addition of non-adsorbing polymers to a colloidal suspension can induce an effective depletion attraction between the colloidal particles[1], leading to a much richer phase behavior [2, 3] than that normally observed for atomic or molecular systems [4]. Colloidpolymer mixtures are therefore often used as model systems of complex fluids, which are normally encountered in technological applications such as food and personal care products, where the attractive interactions may significantly impact product stability. Micron-sized constituent colloids are large enough to be imaged with light, while still small enough to have their dynamics driven by $k_{B} T$, the thermal energy, where $T$ is the temperature and $k_{B}$, the Boltzmann constant. Both the range and magnitude of the attractive interactions can by finely tuned by adjusting the radius $r_{p}$ and concentration $c_{p}$ of the added polymers. With sufficiently high $c_{p}$, these mixtures form gels, i.e. networks of particles that span across the volume in a disordered arrested structure, which can sustain shear stresses, despite the small packing fraction $\phi$ occupied by the colloidal particles [5, 6, 7]. In the limit of strong attractions and very small $\phi$, gelation is driven by the diffusion-limited cluster aggregation (DLCA) of fractal clusters of colloidal particles, a mechanism well-studied in the past $[8,9,10]$. In the limit where $\phi$ is large, particles arrest to form colloidal glasses[11], which can be of two fundamental types: repulsive, driven only by packing and geometrical frustration[12], and attractive, when particle 
bonds become dominant, at sufficiently high attraction strength[13, 14]. A simple extension of attractive glasses to lower and lower densities is not necessarily a good explanation for gel formation, due to the intervening of liquid-gas (colloid rich-colloid poor) phase separation. Several different mechanisms have been proposed for the gel transition at low- and intermediate- $\phi$ and high interaction strengths [7]. These include percolation [15, 16], mode-coupling theory (MCT) attractive glass [17], cluster-MCT[18], fluid-crystal transition[19], spinodal-induced gelation[20, 21, 22], viscoelastic phase separation [23]. Stimulated by a series of numerical studies [24, 25, 26] and by analogies with studies of atomic fluids[27] and polymer solutions[28], the hypothesis of arrested spinodal decomposition has recently received a particular attention in experimental studies [29, 30, 31]. One of the greatest challenges in reaching an unambiguous interpretation is to find a way to precisely quantify the attractive interaction potential, which would allow a quantitative connection to be forged between observed experimental phenomena and a well-characterized thermodynamic phase or state.

Thus far, the mapping of the experimental $c_{p}$ into a system-independent thermodynamic parameter has been based on assumptions of a specific shape for the interparticle potential $V(r)$, such as the Asakura-Oosawa (AO) model[1] typically employed to describe colloid-polymer mixtures. Within this model, colloids (with radius $a$ ) behave as hard spheres, while polymers forming coils in solution have a dual nature: with respect other polymers, they are an ideal gas in which polymer coils can interpenetrate; with respect to the colloids, polymers behave as hard spheres of radius $r_{p}$, which cannot interpenetrate the colloidal spheres. With these simple assumptions, an effective colloid-colloid attractive potential is derived,

$$
V_{A O}(r)=k_{B} T \frac{\partial \Pi}{\partial V} F\left(r / 2\left(a+r_{p}\right)\right)
$$

where $\Pi$ is the osmotic pressure, $V$ the volume and $F(x)$ a well defined function[1] which vanishes for $x>1$. The maximum strength of attraction occurs when two colloidal spheres touch, and this attraction is denoted by the parameter $U \equiv V_{A O}(r=a) / k_{B} T$. To facilitate comparison between different experimental conditions, the concentrationdependent radius of the polymer is typically divided by the colloid radius to yield a dimensionless size ratio $\xi \equiv r_{p} / a$. A universal gelation state diagram may then be parameterized by $\phi, \xi$ and $U$, yielding a way to compare different experiments and theories 32 . However, while $\phi$ and $\xi$ are unambiguously defined in experiment, simulation and theory, $U / k_{B} T$ depends entirely on the shape of the interaction potential. This model-dependence of $U$ leaves its quantification vulnerable to the uncertainties in the applicability of a given theory.

Recently Noro and Frenkel 33 . have established a generalized law of corresponding states for systems interacting with short-range attractive potentials $(\xi \lesssim 0.1)$, in terms only of the normalized second virial coefficient $B_{2}^{*} \equiv\left(3 / 8 a^{3}\right) \int_{0}^{\infty}\left(1-e^{-V(r) / k_{B} T}\right) r^{2} d r$. For a given value of $B_{2}^{*}$, any system interacting with any given short-ranged attractive potential (e.g. AO, square well or generalized Lennard-Jones) shares the same thermodynamic properties and structure. Hence, either a direct measurement of $B_{2}^{*}$ 
or a connection of the experimental attraction strength (e.g. expressing $c_{p}$ in terms of $B_{2}^{*}$ ) would allow a universal description of the system. For example, one recent work on lysozyme has used the Noro-Frenkel law to express the crystallization transition under various solvent conditions in terms of a universal $B_{2}^{*}[34]$. Another possibility often exploited in the past is to rely on the well-known Baxter model 35, which corresponds to the sticky hard sphere limit: the attraction range becomes infinitesimal while the attraction strength becomes infinite in such a way that the second virial coefficient $B_{2}$ remains finite. This model is theoretically convenient because it is amenable to analytic treatment within Percus-Yevick (PY) closure 36] and, consequently, it has been used for a long time in comparison to experiments[37]. However, the thermodynamic inconsistency of the PY solution has often led to an inaccurate location of the gel line with respect to the phase-separation line in the thermodynamic phase diagram [38]. Only a recent accurate location of the gas-liquid phase separation, which was obtained numerically by Miller and Frenkel[39, has opened the way for a more careful use of the Baxter model, via the $B_{2}^{*}$ scaling, in relation to experiments where samples were shown to reach equilibrium over the course of several months [30].

Only a truly model-independent measure of the attractive potential, that could be applied to a variety of attractive colloidal systems, would facilitate quantitative comparison between theory and experiments, and help to elucidate the mechanisms driving the onset of gelation. To address this issue, in a recent paper [40, we have introduced a novel method for generic short-range isotropic attractive interactions independent of any specific potential shape or model - that is capable of quantitatively mapping the experimental observations of gel formation in colloid-polymer mixtures onto the corresponding thermodynamic phase diagram. Through the comparison of the cluster mass distributions, measured directly at the single-particle level using confocal microscopy and in simulations of various short-ranged attractive potentials, we have established a one-to-one relationship between the experimental $c_{p}$ and $B_{2}^{*}$, building on the Noro and Frenkel law 33 . In doing so, we provide unambiguous evidence that gelation takes place precisely where spinodal decomposition occurs, evidencing a strong link between thermodynamic behaviour and dynamic arrest. The gel is triggered from the initial separation into two phases, of which the densest may become locally arrested due to an intervening attractive glass transition[7], and hence kinetics only intervenes after thermodynamics has provided the necessary density fluctuations.

In the present manuscript, we report additional analysis of the experimental system in both the fluid and the gel phase to complement the results previously reported in [40]. These results provide further evidence that gelation in short-ranged attractive colloidpolymer mixtures is an arrested phase separation.

\section{Description of the experimental system}

Sterically-stabilized colloidal spheres of polymethylmethacrylate (PMMA) with DiIC 18 fluorescent dye are suspended in a 5:1 (by mass) solvent mixture of bromocyclohexane 
(CXB, Aldrich) and decahydronaphthalene (DHN, Aldrich) for several months, following procedures already described in [32, 40, 41]. The PMMA particles have radius $a=560 \pm 10 \mathrm{~nm}$, determined with dynamic light scattering. Tetrabutylammonium chloride (TBAC, Fluke) is added until saturation $(\sim 4 \mathrm{mM})$ to screen any residual longrange Coulombic repulsion. The colloidal suspension is carefully buoyancy-matched [40]. Two sets of depletant polystyrene (PS) are used, with molecular weights respectively of $M_{W}=69.2 \mathrm{kDa}$ and $M_{W}=681 \mathrm{kDa}$. From Zimm plots of static light scattering data, the radii of gyration of the two polymers are determined to be 10.0 and $33.0 \mathrm{~nm}$ respectively, yielding $\xi=0.018$ and $\xi=0.059$ in the two cases, always well within the short-range attraction limit where the Noro-Frenkel law is valid. The overlap concentrations, defined as $c_{p}^{*} \equiv 3 M_{W} / 4 \pi r_{p}^{3} N_{A}$, are 27.2 and $7.5 \mathrm{mg} / \mathrm{mL}$, respectively for the two sets of polymers, with $N_{A}$ being the Avogadro's number. In all cases, the raw polymer concentrations are directly measured as the mass ratio of $\mathrm{mg}$ PS per $\mathrm{g}$ of total sample mass, then converted into a $\phi$-dependent free-volume $c_{p}(\mathrm{mg} / \mathrm{ml})[3$. Samples at varying polymer concentration $c_{p}$ are generated while preserving constant $\phi$, although a small decrease is observed with increasing polymer concentration[40]; this may be due to larger clusters sticking to the walls of the macroscopic sample chamber.

Following our previously-reported imaging protocol[32, 40, 41], 3D stacks of 181 images are collected, each an 8-bit TIFF of $1000 \times 1000$ pixels, at 10 frames per second. Each image stack covers a volume of $60 \times 60 \times 60 \mu \mathrm{m}^{3}$, taken from the center of the sample at least $20 \mu \mathrm{m}$ away from any sample chamber surface to minimize edge effects. Each fluid sample is homogenized and equilibrated for four hours, then 26 independent 3D image stacks are collected with an automated confocal microscope[4]. Each gel sample is homogenized and then observed immediately, with 3D stacks of the same sample volume collected every 50 seconds for the first 5000 seconds, then every 1000 seconds for the subsequent 100,000 seconds.

Using confocal microscopy, we observe and individually locate each colloidal particle in the sample imaging volume in 3D; a typical gel is shown in Fig. 1. Most of the colloidal particles are connected in a spanning network, while at all times a residual fraction of particles is present in monomers and smaller clusters that freely diffuse throughout the voids of the network. We also observe a continuous exchange between these unconnected particles and the gel network, due to the finite attraction strength of order a few $k_{B} T$. However, once a percolating gel network forms (which happens generally within a few thousand seconds), it persists for all experimentally observed time - it is arrested.

Locating each particle individually with confocal microscopy also allows more detailed structural analysis, such as a direct calculation of the static structure factor, defined as $S(q) \equiv\left\langle\left|\sum_{i=1}^{N} e^{i \mathbf{q} \cdot \mathbf{r}_{\mathbf{j}}}\right|^{2}\right\rangle / N$. For fluid samples, we average over the 26 independent configurations. For gel samples, we follow a single configuration over time. We calculate $S(q)$ for a cubic box of size $50 \mu \mathrm{m}$, centered within the sample imaging volume, in order to minimize edge effects, which if present, will only affect the range $2 q a \lesssim 0.2$.

This precise way to determine the static structure factor allows a very stringent 


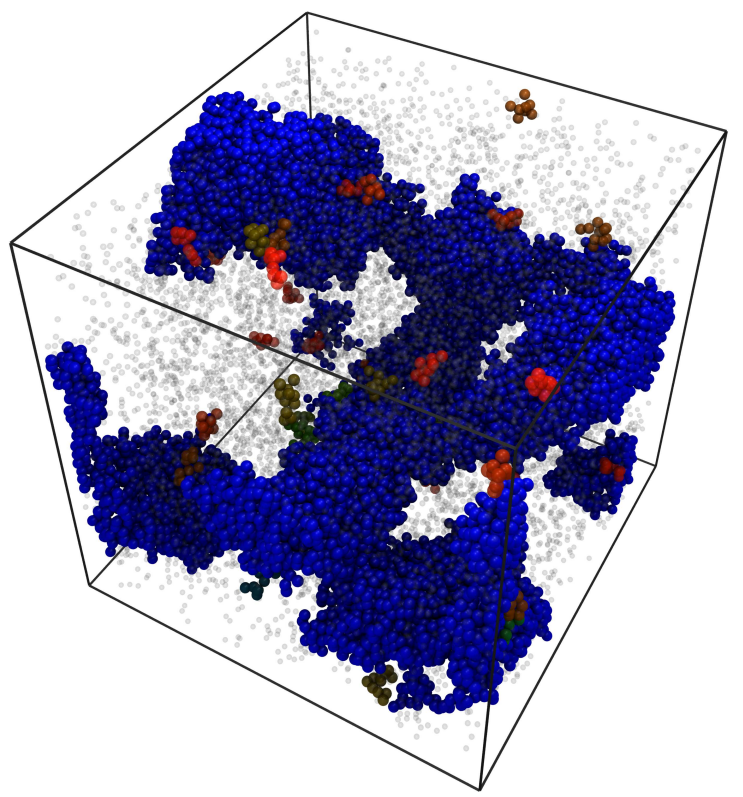

Figure 1. 3d-Reconstruction of the gel for $\phi=0.14, \xi=0.059$ and $c_{p}=1.80 \mathrm{mg} / \mathrm{ml}$. Isolated clusters are shown in different colors for different cluster sizes, while monomers are depicted as transparent (and smaller) spheres.
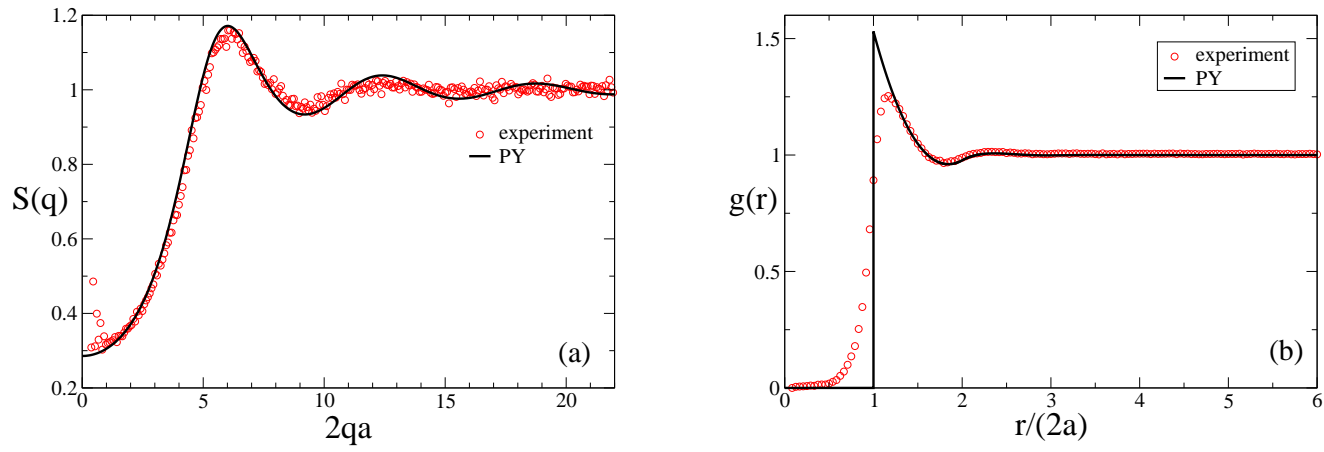

Figure 2. For $\phi=0.16$ and $c_{p}=0$, (a) static structure factor and (b) direct correlation function, calculated using the confocal microscopy coordinates (circles), compared to the PY calculations for hard spheres at the same $\phi$ (full lines).

test on how close our colloids are to being a model hard-sphere system. In Fig[2(a), 
we plot $S(q)$ for an experimental sample with $\phi=0.16$ and $c_{p}=0$, together with PY theoretical predictions at the same $\phi$. The comparison also permits an estimate of the particle radius which best reproduces the superposition of the peak positions, which in this case turns out to be $\simeq 600 \mathrm{~nm}$, quite close to the one measured with dynamic light scattering. We observe a remarkable agreement of $S(q)$ with the theoretical predictions essentially over all length-scales, which demonstrates that, in the absence of polymer, our colloids behave as hard spheres. Similar results are found for the other investigated $\phi$ values. We also examine the direct correlation function $g(r)$, the Fourier transform of $S(q)$ [36]. Experimental data and the PY prediction are shown for the same sample in Fig 2(b). Because these fluid particles diffuse while being imaged, their motion provides a fundamental physical limit on how precisely their centers can be located. Our uncertainty from the image-processing software is well under $50 \mathrm{~nm}$ in all directions [42]. This positional uncertainty smears out the shape of $g(r)$, especially close to the contact peak, resulting in an apparent worse agreement with the PY solution. We therefore conclude that the $S(q)$, whose signal is maximum around $q a \approx \pi$, is a better observable for direct quantitative comparison between confocal images and theory/simulations.

\section{Locating the gel transition onto the thermodynamic phase diagram}

To locate the gel transition on the thermodynamic phase diagram, we have employed a method based on the comparison of experimentally-determined cluster mass distributions with those obtained from simulations in the fluid phase up to gel point [40]. For each experimental sample (with a well-defined $c_{p}$ ), its cluster mass distribution was determined, after defining as connected any pair of particle whose distance is less than $1.3 \mu \mathrm{m}$. The choice of this value, which is larger than the range of attraction, allows us to minimize spurious effects introduced by the aforementioned particle position uncertainties. Simulations were run at the same value of $\phi$ and several values of $B_{2}^{*}$; in order to identify the optimal $B_{2}^{*}$ value, the cluster mass distribution that best matches the experimental data is identified via a least-squares minimization. Connected particles were defined with the same procedure in both experiments and simulations. There are no free parameters involved in this matching procedure, which uniquely associates each experimental $c_{p}$ with a theoretical value of $B_{2}^{*}$. Because the experimental conditions are in the short-ranged attractive limit, we can in principle use any short-ranged attractive potential in the simulations. By using several potential shapes (AO, square well and generalized Lennard-Jones) - all of which yield quantitatively identical results [40]—we provide stringent reconfirmation of the validity of the Noro-Frenkel law.

The results of this $c_{p}-B_{2}^{*}$ mapping procedure are presented in Fig. 3 for $\phi=$ 0.045 and the two studied sets of polymers described above. Horizontal lines mark the experimentally-observed concentration threshold $c_{p}^{g e l}$ denoting the transition from fluid to gel for each polymer. The vertical line denotes the boundary of spinodal decomposition expressed as a value of $B_{2}^{*}$, which depends only on $\phi$. This boundary is estimated both within simulations, through the monitoring of the time-dependence of 
Gelation as arrested phase separation in short-ranged attractive colloid-polymer mixtures7

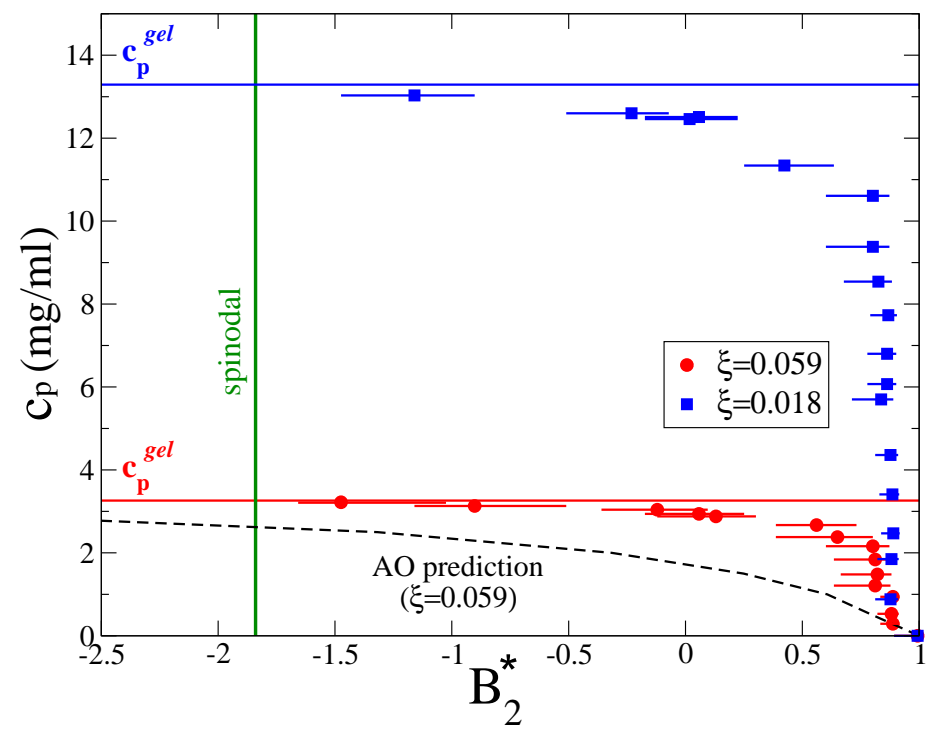

Figure 3. Dependence of polymer concentration on the thermodynamic universal parameter $B_{2}^{*}$ for $\phi=0.045$ and two sets of polymers, with $\xi=0.018$ and $\xi=$ 0.059. The experimental gel transition is observed to happen exactly at the onset of thermodynamic phase separation. The dashed line is the AO prediction for $\xi=0.059$.

the energy and of the small angle static structure factor [24], and via the PY energy route [36], which has been shown to be quite accurate for predicting the spinodal temperature [39]; both values coincide within the error bars. Without exception, the data reported in Fig. 3, as well as those for other studied $\phi$ (not shown here) [40], demonstrate that the gel transition takes place right at the onset of spinodal decomposition. (Note that the $B_{2}^{*}$ scale is rather expanded when compared to a temperature scale, so that the observed distance from the last fluid sample to the gel boundary is very small, and comparable to the experimental error in $c_{p}$.) These data suggest a mechanism for gelation of short-ranged attractive particles: the system first enters the unstable region, where density fluctuations drive the formation of locally-dense liquid droplets; then these denser regions arrest probably through an intervening attractive glass transition.

Fig. 3 provides the 'true' relationship between polymer concentration and thermodynamic parameters: given the correct potential model to describe the system, its $B_{2}^{*}$ should superimpose onto these points. However, since $B_{2}^{*}$ is an integrated quantity of the potential in terms of particle distance, the original potential cannot be recovered easily from $B_{2}^{*}$ alone. However, if the simple AO formula (Eq.1) is used to determine $B_{2}^{*}$ as a function of $c_{p}$ (dashed lines in Fig. 3), the resulting relation is rather different from what the cluster mass distribution mapping predicts. To reach any given $B_{2}^{*}$, the $\mathrm{AO}$ model predicts a much smaller value of $c_{p}$ than that observed in experiment, demonstrating that it does not quantitatively describe the potential for our experimental systems. The discrepancy, as expected, becomes larger and larger for increasing polymer concentrations. 
Gelation as arrested phase separation in short-ranged attractive colloid-polymer mixtures 8

\section{Characterization of the Gel States}

We now examine some features of the observed gel states. For each studied $\phi$, we have monitored the evolution over time of several gel states at different $c_{p}$. In Ref.[40] we have reported $S(q)$, obtained from the particle coordinates, across the fluid-gel transition. We observed that while the fluid, even at the highest $c_{p}$, retains a low value of $S(q \rightarrow 0)$, a big jump of about two orders of magnitude in the low- $q$ peak of $S(q)$ is found as soon as the system crosses to a gel state. Moreover, we followed the evolution in time of this spinodal peak, finding an initial slow coarsening process (compatible with a power law $t^{-1 / 6}$ ), later interrupted by arrest, i.e. at long times the structure of the system reaches a final state and remains subsequently unchanged [40]. These results are in good agreement with numerical simulation studies in the arrested spinodal region 24, 25].

We here complement these results with the additional evidence that the arrested structure crucially depends on the location of the studied state point within the twophase region. Indeed, we plot in Fig, 4 (a) and (b) the evolution of $S(q)$ with time for $\phi=0.16$ and two different $c_{p}$ values. On the left panel, the data correspond to a system which is just inside the gel boundary, i.e. a (shallow) quench just within the spinodal region. In this case, the low- $q$ peak of $S(q)$ undergoes quite a significant coarsening process, growing in amplitude by almost two orders of magnitude, before eventually arrest takes place and the growth stops at long times. On the right panel, we report data corresponding to a much deeper quench inside the spinodal region. Here, the coarsening process almost does not proceed at all, and the structure stops changing quite rapidly, without even developing a large compressibility. Also the peak position does not move significantly with time. These results show that the resulting gels formed by arrested spinodal can differ very much in their final structure, and in particular in their mesh size and compressibility, depending on the chosen quench path inside the two-phase region.

We also monitor the evolution of $S(q)$ using the scaling form for spinodal-driven aggregation derived by Furukawa 43,

$$
S\left(q / q_{1}, t\right) \sim q_{1}(t)^{-d} F\left(q / q_{1}\right),
$$

which was also generalized to the case of fractal clusters undergoing DLCA by Carpineti and Giglio[10]. Here as typical length scale we use $q_{1}(t) \equiv$ $\left(\int_{0}^{q_{c}} S(q, t) q d q\right) /\left(\int_{0}^{q_{c}} S(q, t) d q\right)$ where $q_{c}=6 a$ is an appropriate cut-off value, which provides the first moment of the wave-vector in the low- $q$ peak of $S(q)$ to reduce numerical noise, while $F\left(q / q_{1}\right)$ is a time-independent scaling function and $d$ is the spatial dimension. For fractal aggregation this is found to be replaced by the fractal dimension of the clusters[10]. Within our time window of observation of the gel states, three different time intervals can be identified: an early stage (which we observe only partially, as our first observation time is equal to 100s), an intermediate regime where the Furukawa scaling should hold; and, finally, the late stage or arrested regime, where the system structure does not evolve any longer. In order to visualize the intermediate regime and to demonstrate the scaling of $\mathrm{Eq} 2$ with $d=3$, we focus on state points not 

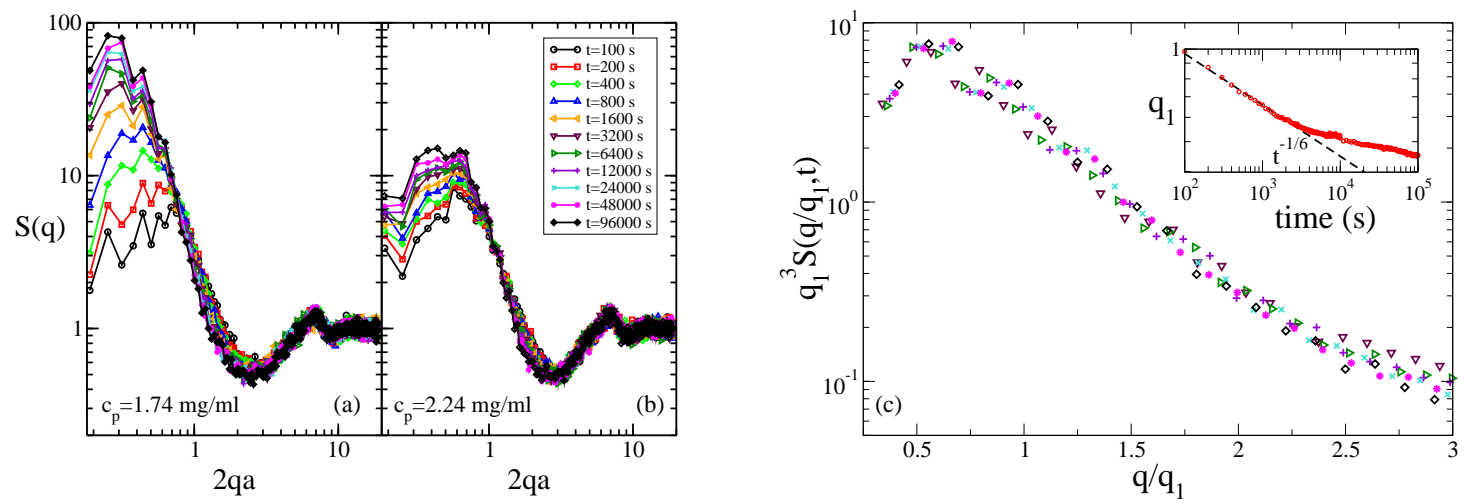

Figure 4. (a) Shallow versus (b) deep quench evolution in time of $S(q)$ for $\phi=0.16$. (c) Scaling of $S(q)$ as in [10] for the shallow quench case reported in (a) and $t \gtrsim 3000 s$. The inset shows the time dependence of $q_{1}$ for the same state point; at short times the reduction of the characteristic length scale is compatible with $t^{-1 / 6}$, later followed by a slower decrease. Symbols are consistent throughout all panels.

too far from the spinodal boundary, where we observe a significant evolution of $S(q)$ over time. We find that our data, under these shallow quench conditions, do scale onto a single master curve at intermediate times, well before arrest takes place, as predicted by the scaling. Trivially, data from later times also scale onto the same master curve. As an example we show in Fig.4(c) the scaled data corresponding to the shallow quench of Fig.4(a). For this particular state point, a significant evolution of $S(q)$ over time is observed, as it is evident from the time-dependence of $q_{1}$ reported in the inset. For $t \gtrsim 3000 s$, which interestingly appears to be very close to the time where $q_{1}$ departs from the $t^{-1 / 6}$ law towards a slower form (until saturation, not observed just for this particular sample), the scaling holds at all subsequent times. For all other studied state points, arrest takes place much earlier so that the scaling can be demonstrated only in a narrower regime. In all cases, the scaling does not providence evidence of a fractal structure of the clusters.

Another measure of the inhomogeneous character of the arrested states is the so-called demixing (or inhomogeneous) parameter $\Psi_{n}$ 444, which can be evaluated by dividing the system into $n^{3}$ boxes and measuring the local density in each box. Hence,

$$
\Psi_{n} \equiv \sum_{k=0}^{n^{3}}\left|\rho_{k}-\rho\right|^{2},
$$

where $\rho_{k}$ is the density in the $k$-th box and $\rho=3 \phi /\left(4 \pi a^{3}\right)$ is the average particle number density. In a fluid or homogeneous state, this parameter averages to zero, while as soon as inhomogeneities appear, it grows. A comparison of $\Psi_{n}$ for different attraction strengths can provide information on how the degree of inhomogeneity varies with different quench paths. We show results for $\Psi_{4}$-calculated by dividing the imaged volume of $(52 \mu \mathrm{m})^{3}$ into $4^{3}=64$ boxes of $13 \mu \mathrm{m}$ edge length — versus time, in Fig.5(a) for 
$\phi=0.045$ and all studied quench depths. We also repeated our analysis for a smaller box edge (of $4 \mu \mathrm{m}$ ), finding the same qualitative results. The evolution in time of $\Psi_{4}$ for all gel samples displays an initial growth followed by a plateau region, similar to what observed for the maximum height of the low- $q$ peak of $S(q)$, named $S^{M A X}(q)$, for the same state points, reported in Fig.5(b). In the inset of Fig.5(a) the average (for fluid states) and
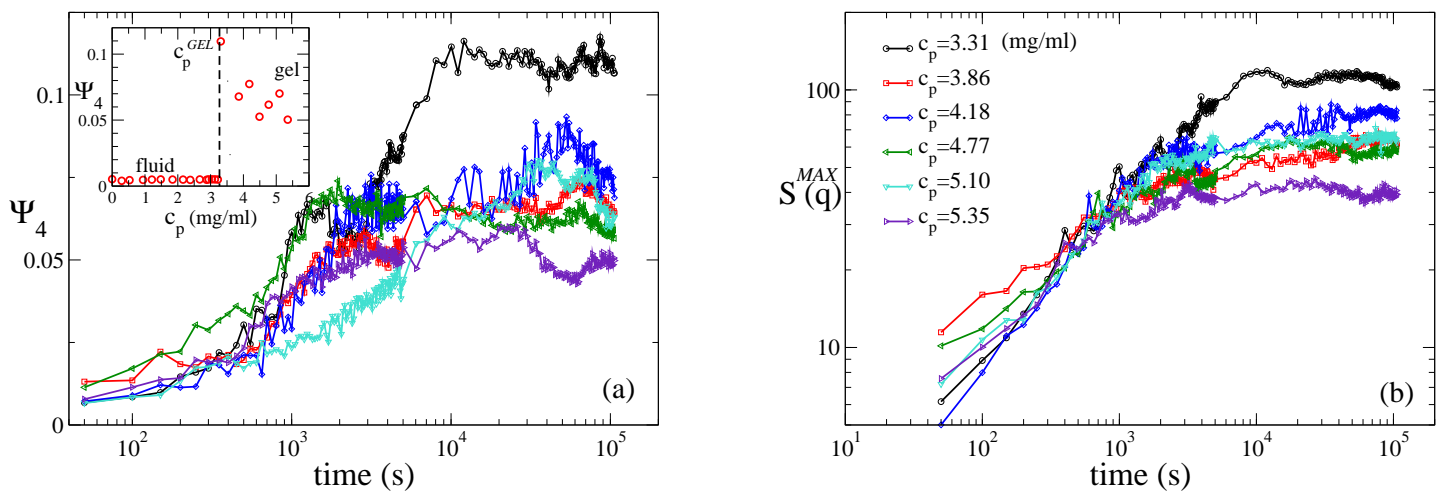

Figure 5. (a): Demixing parameter $\Psi_{4}$ evolution with time for the gel states with $\phi=0.045$. Inset: the fluid to gel transition in terms of $\Psi_{4}$; (b) $S^{M A X}(q)$, the maximum height of $S(q)$, evolution with time for the same state points. Identical lines and symbols are used in both panels.

long-time limiting value (for gel states) of $\Psi_{4}$ is reported, in order to show, once again, the crossing of the spinodal region in correspondence of the gel transition. It is intructive to notice that, apart from the gel state very close to the spinodal boundary (i.e. with $c_{p}=3.31 \mathrm{mg} / \mathrm{ml}$ ), whose spinodal decomposition process seems to proceed further than the others before finally arresting, all other gel states, corresponding to deeper quenches, seem to converge toward a similar value of the inhomogeneity parameter. Similar results are also found for $\phi=0.16$.

Finally, we examine the local density (or $\phi$ ) distribution for several values of the box size. This approach is sometimes implemented in simulations to establish the presence of a two-phase region [45]: if two distinct peaks appear, then two phases of different densities can be properly identified. The observation of the two peaks is strongly connected to the choice of a box size comparable to the size of the spatial density fluctuations, and requires a significant evolution of the phase separation process to be observed 45]. Fig. 6 shows the evolution in time of $P(\phi)$ for two different box sizes, respectively $L=3 \mu \mathrm{m}$ and $L=5 \mu \mathrm{m}$ for $\phi=0.045$ (corresponding to an average number of particle per box of respectively 1.5 and 7.1). Results show that the system starts from a rather homogeneous condition (since $P(\phi)$ on the $L=5 \mu \mathrm{m}$ scale shows a maximum around the average $\phi$ ), then evolves in the direction of increasing the density fluctuations (since states with both small and large packing become preferentially probed) until arrest is reached. On the small $L=3$ scale, the local density increases even more than a factor 
15, reaching values comparable to a dense packing of spheres $(\phi>0.6)$, consistent with the estimates of the internal gel density derived in Ref. [40]. Smaller $\phi$ values are probed on the $L=5$ scale, suggesting that the characteristic mesh of the aggregate is comparable to the smaller box size. We also note that, in the time window preceding dynamic arrest, we do not observe the formation of a bimodal distribution, suggesting that gel formation may precede true bulk phase separation.
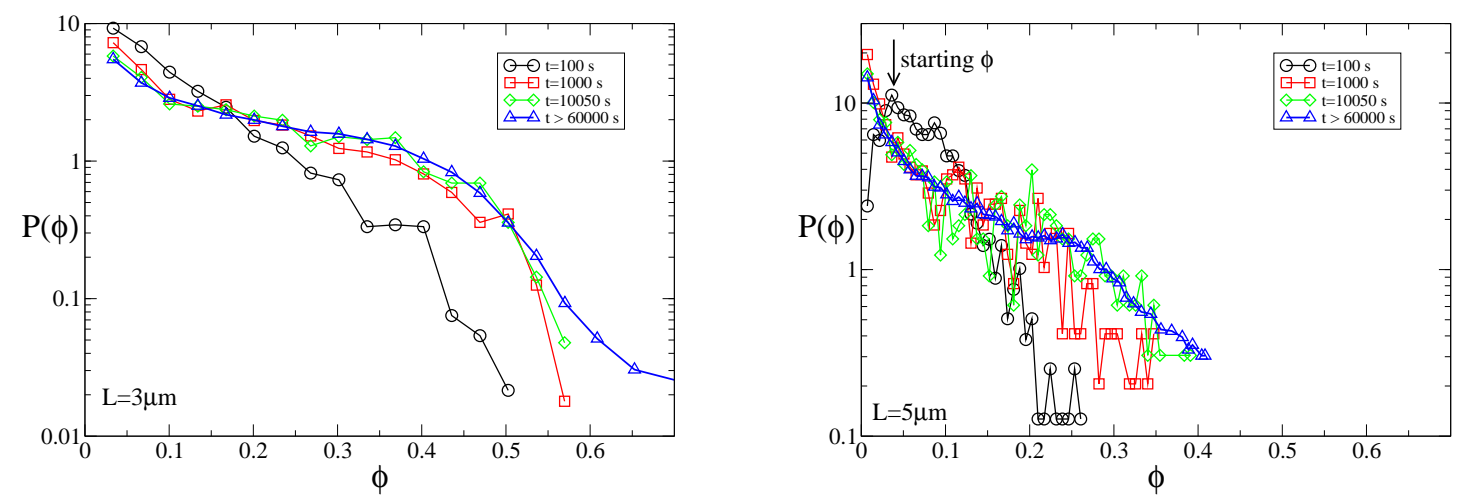

Figure 6. Evolution in time of local packing fraction probability $P(\phi)$, obtained measuring the local $\phi$ in small boxes of sizes 3 (left) and 5 (right) $\mu \mathrm{m}$ ), for the state point $\phi=0.045$ and $c_{p}=3.31 \mathrm{mg} / \mathrm{ml}$.

\section{Conclusions}

In this manuscript, we have provided additional evidence of the mechanism at the origin of the gel transition in short-ranged attractive colloid-polymer mixtures. We have firstly shown that the experimental colloidal system is well characterized: without added polymers, it behaves as a system of nearly-perfect hard spheres. Then, comparing cluster distributions in experiments and simulations has allowed us to precisely map the experimental gel transition on the thermodynamic phase diagram, quantifying the attraction strength in terms of the normalized second virial coefficient $B_{2}^{*}$. This universal parameter contains all the necessary information for short-ranged isotropic attractive potentials to entirely determine the structure and connectivity properties of the system. In this way, our procedure is independent of any specific choice of interaction potential model and is relevant to any short-ranged isotropic attractive system. Our findings unambiguously show that thermodynamic phase separation triggers the kinetic arrest 40 .

A growing consensus has recently favored the arrested phase separation mechanism for gelation in these short-ranged attractive systems. A number of simulations studies [24, 25, 26] and various experimental colloid investigations - including colloids with depletion interactions [29, 30] as well as mixtures of oppositely charged 
colloids 46] - all support this picture, which may also be relevant for atomic glassformers 27]. Moreover, our results confirm important conclusions drawn from data on attractive lysozyme solutions, recently reported by Cardinaux et al. 31]. Both in our colloidal system and in the protein solution case, gelation is caused by the arrest of the dense phase induced by the liquid-gas spinodal decomposition. Yet differences between the two systems appear to arise in the estimated value of the gel packing fraction; this packing fraction appears to be quench-depth independent as evaluated in Ref. [40] and further confirmed by the present analysis, while dramatically depending on quench depth in lysozyme, where the density of the dense phase shows a marked decrease with increasing attraction strength[31]. However, several factors may contribute to these reported differences. First, differences in the interaction potential are present between lysozyme and colloid-polymer mixtures. In lysozyme, electrostatic repulsion[47] can play an important role and, in principle, anisotropy effects (due to shape or patchyness) could be relevant. Indeed, one observes a rather low- $\phi$ position of the critical point ( $\phi_{c} \approx 0.18[31]$ ) as compared to standard short-ranged attractive potentials $\left(\phi_{c} \simeq 0.27[48]\right.$. Second, the methods used to establish the local gel volume fraction are rather different in the two cases. While we have used a microscopic method, based on single-particle location, to estimate the internal volume fraction of the gel [40], Cardinaux et al. determine the gel volume fraction by centrifuging the lysozyme solution after the phase separation was arrested, decanting the supernatant, and measuring the resulting macroscopic volume fraction. Whether these two metrics would yield the same results, if they could both be performed on the same sample, it is presently unknown. A further complication arises by the fact that single $3.4 \mathrm{~nm}$ lysozyme proteins cannot be individually resolved. Finally, the finite size of the imaged volume in our experiment, as compared to the macroscopic volume (compared to particle size) accessed in [31, could also be used as an explanation for the discrepancy, although we performed additional checks for larger samples of millimetric size confirming our previous findings.

Another interesting question relates to why we do not observe any state point undergoing a complete gas-liquid phase separation within our study. In all experimentally studied cases for samples that begin to phase separate, we find that a gel forms at long times. Indeed, for such short-ranged attractive systems at the values of $\phi$ that we study, the difference in attraction strength that would allow to explore the liquid region is very small and roughly comparable to the experimental error in $c_{p}$. However, we do observe some state points, very close to the spinodal boundary where arrest only arises at very long times (see Fig 4). Also, it appears that our studied state points always refer to quenches inside the spinodal, where the liquid droplets rapidly coalesce and form an arrested spanning network. Another interesting case would be to look at quenches inside the region at low $\phi$ delimited between binodal and spinodal lines, where unconnected clusters may become arrested, forming the so-called glassy 'beads' [18, 49].

Finally, we discussed in some detail the properties of the observed gel states. We have provided evidence, from complementary types of analysis, that the resulting states 
are rather inhomogeneous, that their final structure depends on the quench path, and that locally the internal gel volume fraction is very high [40]. Kinetic arrest intervenes before phase separation has proceeded enough to allow the visualization of two distinct peaks in the local density distribution [45], and the system becomes trapped in a highly heterogeneous state. Future work will be devoted to attempts to validate our method at larger packing fractions and to test its limit of validity with increasing range of attraction.

\section{Acknowledgments}

EZ and PJL equally contributed to the present work. We thank A. B. Schofield for the synthesis of the PMMA particles and P. Schurtenberger and E. Sanz for useful comments. We acknowledge support from Marie Curie Network on Dynamical Arrest of Soft Matter and Colloids MRTN-CT-2003-504712, the NoE SoftComp NMP3-CT2004-502235, NASA (NAG3-2284), the NSF (DMR-0602684) and the Harvard MRSEC (DMR-0820484).

\section{References}

[1] Asakura S and Oosawa F 1958 J. Polym. Science 33 183-192

[2] Ilett S M, Orrock A, Poon W C K and Pusey P N 1995 Phys.Rev E 51 1344-1352

[3] Lekkerkerker H N W, Poon W C K, Pusey P N, Stroobants A and Warren P B 1992 Europhys. Lett. 20(6) 559-564 414

[4] Anderson V J and Lekkerkerker H N W 2002 Nature 416 811-815

[5] Trappe V and Sandkühler P 2004 Curr. Op. Coll. Interf. Sci. 8 494-500

[6] Cipelletti L and Ramos L 2005 J. Phys.:Condens. Matter 17253

[7] Zaccarelli E 2007 J. Phys.: Condens. Matter 19323101

[8] Vicsek T 1989 Fractal Growth Phenomena (Singapore: World Scientific)

[9] Weitz D A, Huang J S, Lin M Y and Sung J 1985 Phys. Rev. Lett. 54(13) 1416-1419

[10] Carpineti M and Giglio M 1992 Phys. Rev. Lett. 68(22) 3327-3330

[11] Sciortino F and Tartaglia P 2005 Adv. in Phys. 54471

[12] Pusey P N and van Megen W 1987 Phys. Rev. Lett. 59(18) 2083-2086

[13] Dawson K A, Foffi G, Fuchs M, Götze W, Sciortino F, Sperl M, Tartaglia P, Voigtmann T and Zaccarelli E 2001 Phys. Rev. E 63011401

[14] Pham K N, Puertas A M, Bergenholtz J, Egelhaaf S U, Moussaïd A, Pusey P N, Schofield A B, Cates M E, Fuchs M and Poon W C K 2002 Science 296 104-106

[15] Grant M C and Russel W B 1993 Phys. Rev. E 47 2606-2614

[16] Shah S A, Chen Y L, Schweizer K S and Zukoski C F 2003 J. Chem. Phys. 119(16) 8747-8760

[17] Bergenholtz J, Poon W C K and Fuchs M 2003 Langmuir 19 4493-4503

[18] Cates M E, Fuchs M, Kroy K, Poon W C K and Puertas A M 2004 J. Phys.:Cond. Matt 164861

[19] Pusey P N, Pirie A D and Poon W C K 1993 Physica A 201 322-331

[20] Verhaegh N A M, Asnaghi D, Lekkerkerker H N W, Giglio M and Cipelletti L 1997 Physica A 242 104-118

[21] Poulin P, Bibette J and Weitz D A 1999 Eur. Phys. J. 7 277-281

[22] Sciortino F, Bansil R and Stanley E 1993 Phys. Rev. E 47 4615-4618

[23] Tanaka H 2000 J. Phys.:Cond. Matt 12207

[24] Zaccarelli E, Sciortino F, Buldyrev S V and Tartaglia P 2004 Short-ranged attractive colloids: What is the gel state? (Elsevier, Amsterdam) pp 181-194 
Gelation as arrested phase separation in short-ranged attractive colloid-polymer mixtures14

[25] Foffi G, De Michele C, Sciortino F and Tartaglia P 2005 J. Chem. Phys. 1224903

[26] Charbonneau P and Reichman D R 2007 Phys. Rev. E 75011507

[27] Sastry S 2000 Phys. Rev. Lett. 85 590-593

[28] Leone M, Sciortino F, Migliore M, Fornili S L and Vittorelli M B P 1987 Biopolymers 26 743-761

[29] Manley S, Wyss H, Miyazaki K, Conrad J, Trappe V, Kaufman L J, Reichman D R and Weitz D A 2005 Phys. Rev. Lett. 94238302

[30] Buzzaccaro S, Rusconi R and Piazza R 2007 Phys. Rev. Lett. $99098301-+$

[31] Cardinaux F, Gibaud T, Stradner A and Schurtenberger P 2007 Phys. Rev. Lett. 99 118301-+

[32] Lu P J, Conrad J C, Wyss H M, Schofield A B and Weitz D A 2006 Phys. Rev. Lett. 96028306

[33] Noro M G and Frenkel D 2000 J. Chem.Phys 113 2941-2944

[34] Sedgwick H, Cameron J E, Poon W C K and Egelhaaf S U 2007 J. Chem. Phys. $127125102-+$

[35] Baxter R J 1968 J. Chem. Phys. 492770

[36] Hansen J P and MacDonald I R 2006 Theory of Simple Liquids 3rd ed (London: Academic)

[37] Chen S H, Rouch J, Sciortino F and Tartaglia P 1994 J. Phys.: Condens. Matter 6 10855-10883

[38] Verduin H and Dhont J K G 1995 J. Coll. Inter. Science 172 425-437

[39] Miller M A and Frenkel D 2003 Phys. Rev. Lett. 90(13) 1-4

[40] Lu P J, Zaccarelli E, Ciulla F, Schofield A B, Sciortino F and Weitz D A 2008 Nature 453 499-503

[41] Lu P J, Sims P A, Oki H, Macarthur J B and Weitz D A 2007 Opt. Express 158702

[42] Gao Y and Kilfoil M 2008 Opt. Express (submitted)

[43] Furukawa H 1985 Adv. Phys. 34 703-750

[44] Puertas A, Fuchs M and Cates M E 2003 Phys. Rev. E 67031406

[45] Babu S, Gimel J C and Nicolai T 2006 J. Chem. Phys. 125 184512-+

[46] Sanz E, Leunissen M, Fortini A, Dijkstra M and van Blaaderen A 2008 J. Phys. Chem. B 112 10861

[47] Cardinaux F, Stradner A, Schurtenberger P, Sciortino F and Zaccarelli E 2007 Europhys. Lett. 77 48804

[48] Largo J, Miller M A and Sciortino F 2008 J. Chem. Phys. 128 134513-+

[49] Sedgwick H, Kroy K, Salonen A, Robertson M B, Egelhaaf S U and Poon W C K 2005 Eur. Phys. J. E $1677-80$ 